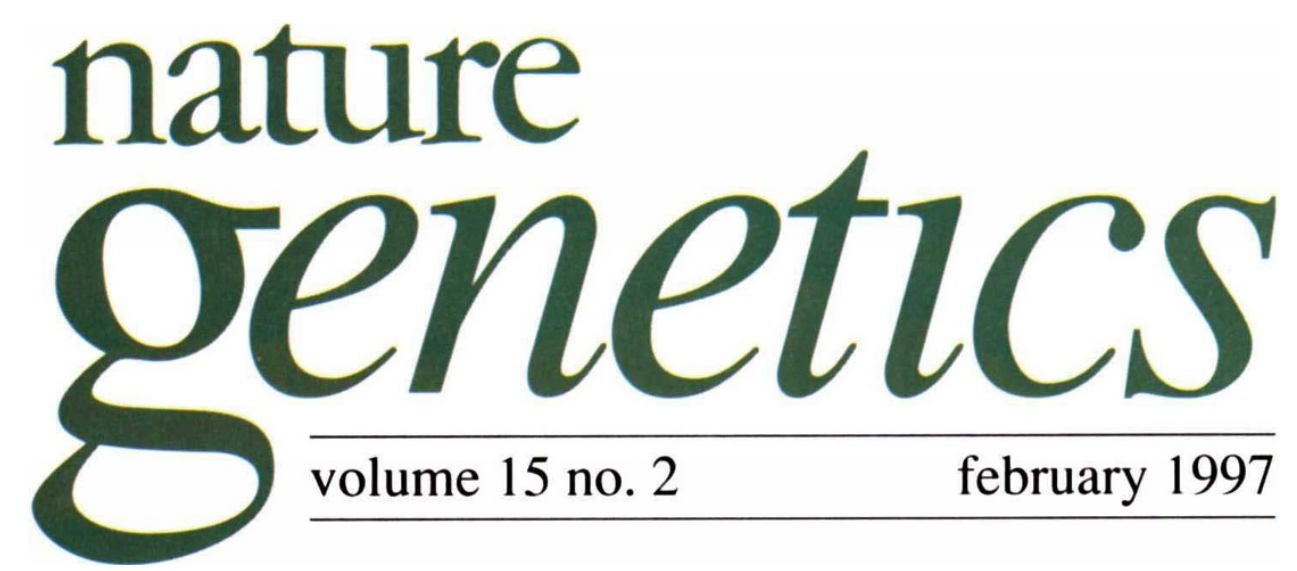

\title{
Entering the total-genomic era
}

With the completion of the sequencing of the yeast genome last year and the sequencing of several other genomes on track, what has been termed 'the postgenomic era' looms ever closer, and questions of what this will mean and where it will lead become paramount. But the phrase somehow suggests that once the sequencing is done, we can all heave a great sigh of relief and never think about the genome again. In reality, the completion of the sequences of the genomes is ushering

"The highest wisdom has but one science - the science of the whole the science explaining the whole creation and man's place in it."

- Leo Tolstoy, War and Peace in a period of more global thinking about what this will really mean for the life sciences. The questions asked and the experiments done will be ever more complex and sophisticated as the information provided is deciphered, catalogued, extracted and ultimately harnessed in a way that will bind all organisms more closely.

A taste of things to come was the meeting held three months ago in Baltimore, Maryland, on "Yeast Genetics and Human Disease"*, whose primary purpose was to stimulate discussion of strategies for using yeast and the information available in the completed yeast genome for greater understanding of the underlying biochemical causes of human disease. The meeting's unusual nature was immediately apparent in that, for its small size, it had a remarkably broad range of talks, including everything from cell-cycle control to genome analysis. Normally a meeting of 200 participants would focus on only one of these topics; however, while several topics were addressed, each session was carefully crafted to include analyses done both in yeast and in mammals. Attendees came away from each session with knowledge of novel data, but also a sense of the differences and similarities of the organisms used, provoking new ways of looking at each data set.

Clearly, the use of a 'simpler' organism to aid understanding of a more 'complex' one is not new. But there are, perhaps, no new ideas, only new ways of implementing them. This meeting represented a conscious effort to bind studies of two organisms into one, not as in the past, with yeast and mammalian geneticists primarily doing their work separately on their gene or system of interest and coming together only in the literature, but to physically bring these groups of researchers together in a retreat-like setting that precipitated a unique set of interactions. The Baltimore meeting raised not so much the question of whether or not these two organisms should be studied together - they 
utilizing multi-organismal methods of study. That is, given that biochemical mechanisms in evolutionarily distant species are far more similar than anyone had originally anticipated and that each organism has unique experimental advantages, the percentage of researchers taking advantage of multi-organismal methods should be far greater. The result of the meeting was an exhilarating experience as those attending became more aware of the synergistic possibilities in such consciously constructed multi-organismal approaches, which will only become more exciting as genome sequences are completed in Caenorhabditis elegans, Drosophila, mice and men.

Ira Herskowitz (Univ. California, San Francisco), in the first talk of the meeting, laid out the ways in which directive use of the yeast system can benefit mammalian studies: i) by using the structural similarity of yeast and human genes, ii) by making functional assays of mammalian products (humanizing yeast) and iii) by cloning human homologues by complementation of yeast mutants. The advent of the completed yeast genome manifestly elevates all of these goals to a new level.

A clear illustration of the importance of capitalizing on the strengths of several organisms is in the work on the cell-cycle protein CDC27 where genetic studies in yeast, cytological studies in human cells and biochemical studies in Xenopus created a larger picture of the mechanisms of cell-cycle control than any alone could pro$v^{\text {vide }}{ }^{1-3}$. In addition, the completion of the yeast genome provides the unique opportunity to characterize and classify by genetic homology and structure whole classes of genes and to compare them to those in other organisms, as was done in the analysis by A. Decottignies and A. Goffeau ${ }^{4}$ (see page 137) of all of the ATP-binding cassette $(A B C)$ proteins in yeast. On a more practical level, the yeast system allows easy differentiation of polymorphisms from mutations in human disease genes as assessed by the ability of different human alleles to transactivate a yeast marker gene ${ }^{5}$ or to complement yeast homologues; a standard system of functional analysis might thus be designed whereby human disease genes are sequenced via DNA chip analy. $\operatorname{sis}^{6}$ and any variants then tested for function in a yeast assay. Beyond this, the straightforward genetics, the relative ease in deciphering components of biochemical pathways, and the potential for reconstituting biochemical systems with homologous genes within yeast are only the beginning of the ways in which directed use of this organism can help in understanding cellular mechanisms. Adding other organisms to the equation only increases complexity of questions that can be asked and answered. As with 'humanizing' the yeast, reconstitution of human systems in the mouse is useful for an entirely different set of questions. One such example is found on page 146 of this issue where A. Jakobovits and colleagues ${ }^{7}$ have reconstituted a component of the human immunoglobulin system, allowing a variety of questions about antibody gene regulation and response to be addressed.

Essentially, the completed sequence of the yeast genome and those soon to follow will allow a straightforward comparison between all organisms: what genes do they have in common, how similar are they, where do they differ and ultimately what experiments can be designed to capitalize on the different strengths of all these organisms to best enhance our understanding of the gene/genes/system of interest. The genomes effectively become the universal language through which all life scientists can communicate ${ }^{8}$. As David Botstein (Stanford Univ.) put it in Baltimore, "the yeast genome is the Rosetta stone." The sequences of all genomes to follow should be written in the same language.

Using other organisms to understand human biology is an old idea. But the Baltimore gathering is one of the first in what will hopefully be many discussions of how to take this idea a step further: how to broaden the scope, and, in effect, to look at complex problems and solve them, as Francis Collins (NIH) stressed, by non-linear means. From all appearances at the meeting, we are not entering the post-genomic era at all, but rather a total-genomic era, where whole genomes and whole organisms generate global ways of thinking and large-scale problem-solving. 